OPEN ACCESS

Edited by:

David Vaudry,

Institut National de la Santé et de la Recherche Médicale (INSERM),

France

Reviewed by:

Barbara Kofler,

University Hospital Salzburg, Austria

Zhaoxiang Bian,

Hong Kong Baptist University,

Hong Kong

*Correspondence:

Jae Young Seong

jyseong@korea.ac.kr

Specialty section: This article was submitted to Neuroendocrine Science, a section of the journal

Frontiers in Neuroscience

Received: 19 February 2019

Accepted: 04 April 2019

Published: 18 April 2019

Citation:

Yun S, Reyes-Alcaraz A, Lee Y-N, Yong HJ, Choi J, Ham B-J, Sohn J-W, Kim D-H, Son GH, Kim H, Kwon S-G,

Kim DS, Kim BC, Hwang J-I and

Seong JY (2019) Spexin-Based

Galanin Receptor Type 2 Agonist for Comorbid Mood Disorders and Abnormal Body Weight.

Front. Neurosci. 13:391

doi: 10.3389/fnins.2019.00391

\section{Spexin-Based Galanin Receptor Type 2 Agonist for Comorbid Mood Disorders and Abnormal Body Weight}

\author{
Seongsik Yun ${ }^{1}$, Arfaxad Reyes-Alcaraz ${ }^{1}$, Yoo-Na Lee', Hyo Jeong Yong ${ }^{1}$, Jeewon Choi ${ }^{2}$, \\ Byung-Joo Ham ${ }^{3}$, Jong-Woo Sohn', Dong-Hoon Kim ', Gi Hoon Son', Hyun Kim', \\ Soon-Gu Kwon ${ }^{4}$, Dong Sik Kim ${ }^{4}$, Bong Chul Kim ${ }^{4}$, Jong-lk Hwang ${ }^{1}$ and \\ Jae Young Seong ${ }^{1 *}$ \\ ${ }^{1}$ Graduate School of Medicine, Korea University, Seoul, South Korea, ${ }^{2}$ Graduate School of Medical Science \\ and Engineering, Korea Advanced Institute of Science and Technology, Daejeon, South Korea, ${ }^{3}$ Department of Psychiatry, \\ College of Medicine, Korea University, Seoul, South Korea, ${ }^{4}$ Neuracle Science Co., Ltd., Seoul, South Korea
}

Despite the established comorbidity between mood disorders and abnormal eating behaviors, the underlying molecular mechanism and therapeutics remain to be resolved. Here, we show that a spexin-based galanin receptor type 2 agonist (SG2A) simultaneously normalized mood behaviors and body weight in corticosterone pelletimplanted (CORTI) mice, which are underweight and exhibit signs of anhedonia, increased anxiety, and depression. Administration of SG2A into the lateral ventricle produced antidepressive and anxiolytic effects in CORTI mice. Additionally, SG2A led to a recovery of body weight in CORTI mice while it induced significant weight loss in normal mice. In Pavlovian fear-conditioned mice, SG2A decreased contextual and auditory fear memory consolidation but accelerated the extinction of acquired fear memory without altering innate fear and recognition memory. The main action sites of SG2A in the brain may include serotonergic neurons in the dorsal raphe nucleus for mood control, and proopiomelanocortin/corticotropin-releasing hormone neurons in the hypothalamus for appetite and body weight control. Furthermore, intranasal administration of SG2A exerted the same anxiolytic and antidepressant-like effects and decreased food intake and body weight in a dose-dependent manner. Altogether, these results indicate that SG2A holds promise as a clinical treatment for patients with comorbid mood disorders and abnormal appetite/body weight.

Keywords: galanin receptor 2 agonist, depression, post-traumatic stress disorder, body weight, appetite, intranasal administration

\section{INTRODUCTION}

Mood disorders such as anxiety and depression are often associated with abnormal eating behaviors, leading to either obesity or a poor diet (Simon et al., 2006), and endocrine and metabolic conditions are both exacerbated in major depression (Simon et al., 2006; Marijnissen et al., 2011). Stress can promote either an increased consumption of palatable and rewarding foods leading to obesity or a diminished appetite causing weight loss (Adam and Epel, 2007), and individuals who 
are underweight or obese are at a high risk for depression and anxiety (Carey et al., 2014). Therefore, mood disorders and abnormal appetite are reciprocally linked (Kloiber et al., 2007). This link has also been observed in animal models of mood disorders (Sharma et al., 2013), suggesting that a common signaling pathway underlies these phenotypes in humans and animals.

Recently, the novel neuropeptide spexin (SPX) and its receptors, galanin (GAL) receptor type $2\left(\mathrm{GAL}_{2}\right.$ receptor), and

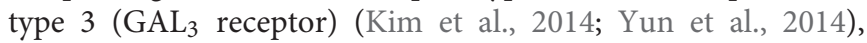
have gained attention for their possible involvement in the bidirectional regulation of mood and feeding behaviors. The function of SPX in feeding behavior is interesting, because it opposes the orexigenic function of GAL, a paralogous neuropeptide (Yun et al., 2015; Furlong and Seong, 2017). Whereas the SPX administration leads to weight loss in dietinduced obese rodents (Walewski et al., 2014) and decreased food intake in goldfish (Wong et al., 2013), GAL leads to an increase in food intake (Karatayev et al., 2009). SPX mRNA levels are markedly decreased in the fat tissues of obese humans (Walewski et al., 2014), whereas the circulating GAL levels, along with neuropeptide $\mathrm{Y}$ and leptin, are significantly higher in obese women (Baranowska et al., 1997). These differences likely reflect the activities of the targeted receptors: SPX binds with high affinity to $\mathrm{GAL}_{2}$ receptor and $\mathrm{GAL}_{3}$ receptor but not $G_{1} L_{1}$ receptor, whereas GAL has high potencies for $G_{1} L_{1}$ receptor and $\mathrm{GAL}_{2}$ receptor but a low potency for $\mathrm{GAL}_{3}$ receptor (Kim et al., 2014). In addition, the $\mathrm{GAL}_{2}$ receptor-mediated downstream signaling induced by SPX and GAL differs. Whereas GAL exerts both $\mathrm{G}_{\mathrm{q}}$ - and $\beta$-arrestin-mediated signaling of $\mathrm{GAL}_{2}$ receptor, SPX shows a biased agonism favoring G-proteinmediated signaling (Reyes-Alcaraz et al., 2018).

When exogenously administered to rodents, GAL increases their immobility in the forced swim test (FST), suggesting an increase in depression-like behavior (Kuteeva et al., 2008). Studies using peptidergic or non-peptidergic compounds with selectivity for GAL receptor subtypes suggested that $\mathrm{GAL}_{1} / \mathrm{GAL}_{3}$ receptormediated signaling contributes to the prodepressive effect, whereas $\mathrm{GAL}_{2}$ receptor-mediated signaling exerts antidepressive effects (Webling et al., 2012). For instance, M617, an agonist of $G_{1} L_{1}$ receptor and $G A L_{2}$ receptor $\left(G A L_{1}\right.$ receptor $>G_{2}$ receptor), induces depression-like behavior, but the nonpeptidergic $\mathrm{GAL}_{3}$ receptor antagonist SNAP37889 decreases anxiety- and depression-like behaviors (Swanson et al., 2005). AR-M1896 (Gal2-11), an agonist of $\mathrm{GAL}_{2 / 3}$ receptor $\left(\mathrm{GAL}_{2}\right.$ receptor $>\mathrm{GAL}_{3}$ receptor), suppresses depression-like behaviors (Webling et al., 2012), but $\mathrm{GAL}_{2}$ receptor knockout mice and $\mathrm{GAL}_{2}$ receptor antagonist M871 injected mice exhibited anxietyand depression-like behaviors (Bailey et al., 2007). Thus, steering SPX action through $\mathrm{GAL}_{2}$ receptor is an optimal approach to simultaneously resolve both mood disorders and abnormal appetite/body weight.

We recently developed SPX-based $\mathrm{GAL}_{2}$ receptor-selective agonists (SG2A) with a much longer half-life in serum than wild-type SPX (Figure 1; Reyes-Alcaraz et al., 2016, 2018). In addition, SG2A, like SPX but unlike GAL, preferentially induced G-protein-mediated signaling over $\beta$-arrestin-dependent

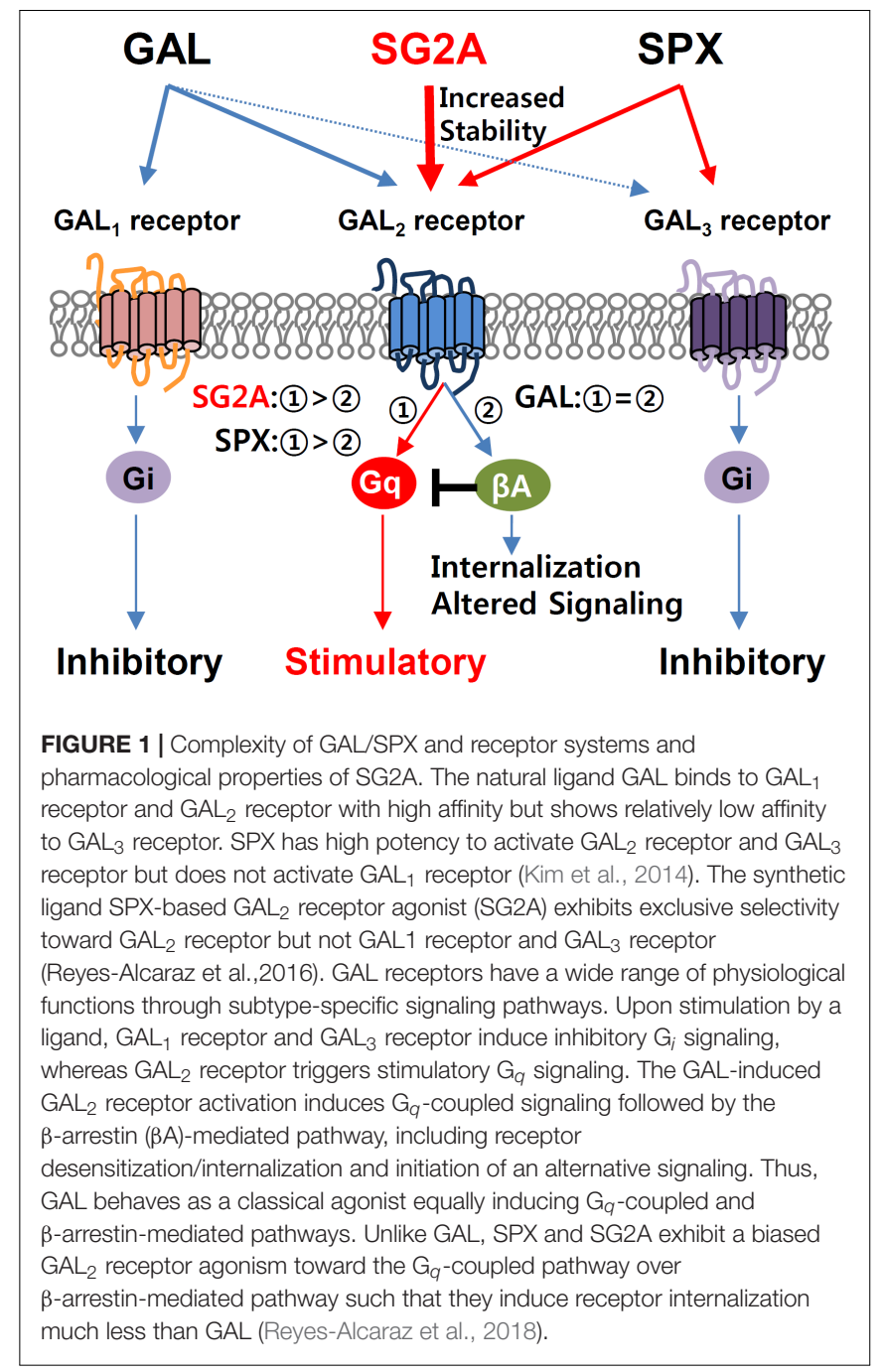

pathway (Reyes-Alcaraz et al., 2018), which avoids the drug tolerance or possible adverse side effects associated with classical agonists (Hausdorff et al., 1990; Yang and Tao, 2017). In the present study, we examined the beneficial effects of intracerebroventricularly (i.c.v.) administered SG2A on body weight and mood changes in mouse models of depression and anxiety disorder and investigated the putative neural networks activated by SG2A. We also examined whether intranasal (i.n.) administration of SG2A produces effects similar to those with i.c.v. administration, which would increase its potential clinical application for patients with comorbid mood disorders and abnormal body weight.

\section{MATERIALS AND METHODS}

\section{Animals}

Wild-type male C57BL/6J mice at 8-weeks old were obtained from Central Lab Animal (Seoul, South Korea) and proopiomelanocortin (POMC)-hrGFP mice were from Jackson Laboratory (\#006421; Bar Harbor, ME, United States). All mice 
were kept in temperature-controlled $\left(22-23^{\circ} \mathrm{C}\right)$ rooms under a 12-h light-dark photoperiod. Standard mouse chow/water were available ad libitum. All animal procedures were approved by the Institutional Animal Care and Use Committee of Korea University (KOREA-2016-0050-C2) and Korea Advanced Institute of Science and Technology (KA2014-02).

\section{Drug Application}

For i.c.v. injections, the mice were anesthetized with a flow anesthesia system, mounted on a stereotaxic apparatus (Stoelting, Wood Dale, IL, United States), and unilaterally implanted with 26-gauge stainless steel guide cannulae (model C315G; Plastics One, Roanoke, VA, United States) in the lateral ventricles [(LV) AP, $-0.55 \mathrm{~mm}$; ML, $1.1 \mathrm{~mm}$; and DV, $-2.05 \mathrm{~mm}$ ]. A 32-gauge dummy cannula was inserted into guide cannula to prevent clogging. Two jewelry screws were implanted into the skull as anchors, and the whole assembly was affixed to the skull with dental cement. Mice were allowed to recover for 2 weeks, singly housed. SG2A (PEG2-NWTdANAALYLFGPdQ-NH 2 ; AnyGen, Gwangju, South Korea) was dissolved in dimethyl sulfoxide (DMSO) to a concentration of $100 \mu \mathrm{M}(170 \mathrm{ng} / \mu \mathrm{l})$ and injected into the $\mathrm{LV}(0.5 \mu \mathrm{l} /$ ventricle $) 2-3 \mathrm{~h}$ before behavioral tests or 1-2 $\mathrm{h}$ before $\mathrm{c}$-fos induction sampling with 33-gauge injector cannulae attached to $10-\mu 1$ Hamilton syringes at a rate of $0.5 \mu \mathrm{l} / \mathrm{min}$. For i.n. administration, a $10 \mathrm{mM}$ stock solution of SG2A dissolved in DMSO was diluted with phosphate-buffered saline (PBS) to 1,3 , and $10 \mu \mathrm{g}$ in $5 \mu \mathrm{l}$ final volume, and $2.5 \mu \mathrm{l}$ of this solution was delivered to each nostril with a pipette.

\section{Behavioral Studies}

Mice were handled for 5 min daily for 3 days before the behavioral tests. Recorded videos were analyzed with an ANY-maze video tracking program (Stoelting).

See Supplementary Information for body weight and food intake measurements, elevated plus maze test (EPMT), open filed test (OFT), tail suspension test (TST), the FST, sucrose preference test (SPT), Pavlovian fear conditioning, unconditioned innate fear response, Y-maze test, and novel object recognition (NOR) test.

\section{Corticosterone Pellet Implantation (CORTI) for the Depression Model}

CORTI was performed as previously described (Demuyser et al., 2016). Mice were randomly assigned to two groups (Sham or CORTI). For the CORTI group, mice were anesthetized with a flow anesthesia system and two slow-release corticosterone pellets (21-day release, $5 \mathrm{mg} /$ pellet; Innovative Research of America, Sarasota, FL, United States) were subcutaneously implanted in the neck to provide an equivalent total exposure of approximately $20 \mathrm{mg} / \mathrm{kg} /$ day corticosterone depending on the weight. Sham group, were treated equally but were not implanted. Cannulae for i.c.v. were then implanted into the LV of animals in both groups. Body weights were recorded every 3 days and every week.

\section{Immunohistochemistry}

Animals were perfused with $4 \%$ paraformaldehyde in PBS, and isolated brains were postfixed in the same fixative overnight. The brains were then cryoprotected in 30\% sucrose, sectioned serially on a cryostat $(40 \mu \mathrm{m})$, and stored in $50 \%$ glycerol $/ 50 \%$ PBS at $-20^{\circ} \mathrm{C}$ until use. Sections were blocked with $10 \%$ horse serum and $0.3 \%$ Triton X-100 for $30 \mathrm{~min}$. Sections were incubated overnight at $4^{\circ} \mathrm{C}$ with primary antibodies against c-fos (Cell Signaling Technology, Danvers, MA, United States), CamKII $\alpha$ (Millipore, Burlington, MA, United States), GAD67 (Millipore), tryptophan hydroxylase [(TPH) Sigma-Aldrich, St. Louis, MO, United States], tyrosine hydroxylase [(TH) SigmaAldrich], NeuN (Millipore), or corticotropin-releasing hormone [(CRH) Peninsula Laboratories, San Carlos, CA, United States]. After several washes with PBS, appropriate secondary antibodies, Alexa 488 and Cy3 with DAPI, were applied for $30 \mathrm{~min}$. The sections were washed, mounted, and observed under a confocal microscope (Leica TCS SP8; Leica Microsystems, Buffalo Grove, IL, United States). For analysis of neurogenesis, mice were administered BrdU [100 mg/kg i.p.; 97\% (+)-5'-bromo-2'deoxyuridine; Sigma-Aldrich] twice per day (8-h interval) for 2 days prior to sacrifice. Before blocking, the brain sections were incubated with $1 \mathrm{~N} \mathrm{HCL}$ for $30 \mathrm{~min}$ at $37^{\circ} \mathrm{C}$ and then incubated overnight with anti-BrdU (Abcam, Cambridge, United Kingdom) and anti-doublecortin [(DCX) Santa Cruz Biotechnology, Dallas, TX, United States] antibodies.

See Supplementary Information for Western blotting, electrophysiology and determination of $\alpha-\mathrm{MSH}$ secretion in POMC neurons.

\section{Statistical Analysis}

The data are presented as means \pm standard errors of the means (SEMs) from at least two independent experiments. Statistical differences between individual groups were evaluated using Student's $t$-tests and/or analyses of variance followed by Newman-Keuls tests as a post hoc comparison. Electrophysiology results were analyzed with Wilcoxon signed-rank tests. Fear memory extinction was evaluated by repeated-measures analyses of variance followed by Bonferroni tests. $P$-value of $<0.05$ was considered statistically significant.

\section{RESULTS}

\section{Effects of SG2A in CORTI Mice}

The effects of SG2A were first addressed in CORTI mice, which exhibit markedly lowered body weights and anhedonia-, anxiety, and depression-like behaviors (Figure 2). The functional phenotype of these mice resembles the hypercortisolism, anhedonia, hypophagia, and weight loss in individuals with melancholic depression (Carroll et al., 2012), according to the Diagnostic and Statistical Manuals of Mental Disorders, 5th edition (American Psychiatric Association, 2013). 14 days after CORTI or sham operations, SG2A were administered i.c.v. for 8 consecutive days. During this time, the SPT was performed first for 2 days, followed by 1-day break, and then the EPMT was performed for 2 days, OFT for 1 day, and TST for 1 day. 3 to 


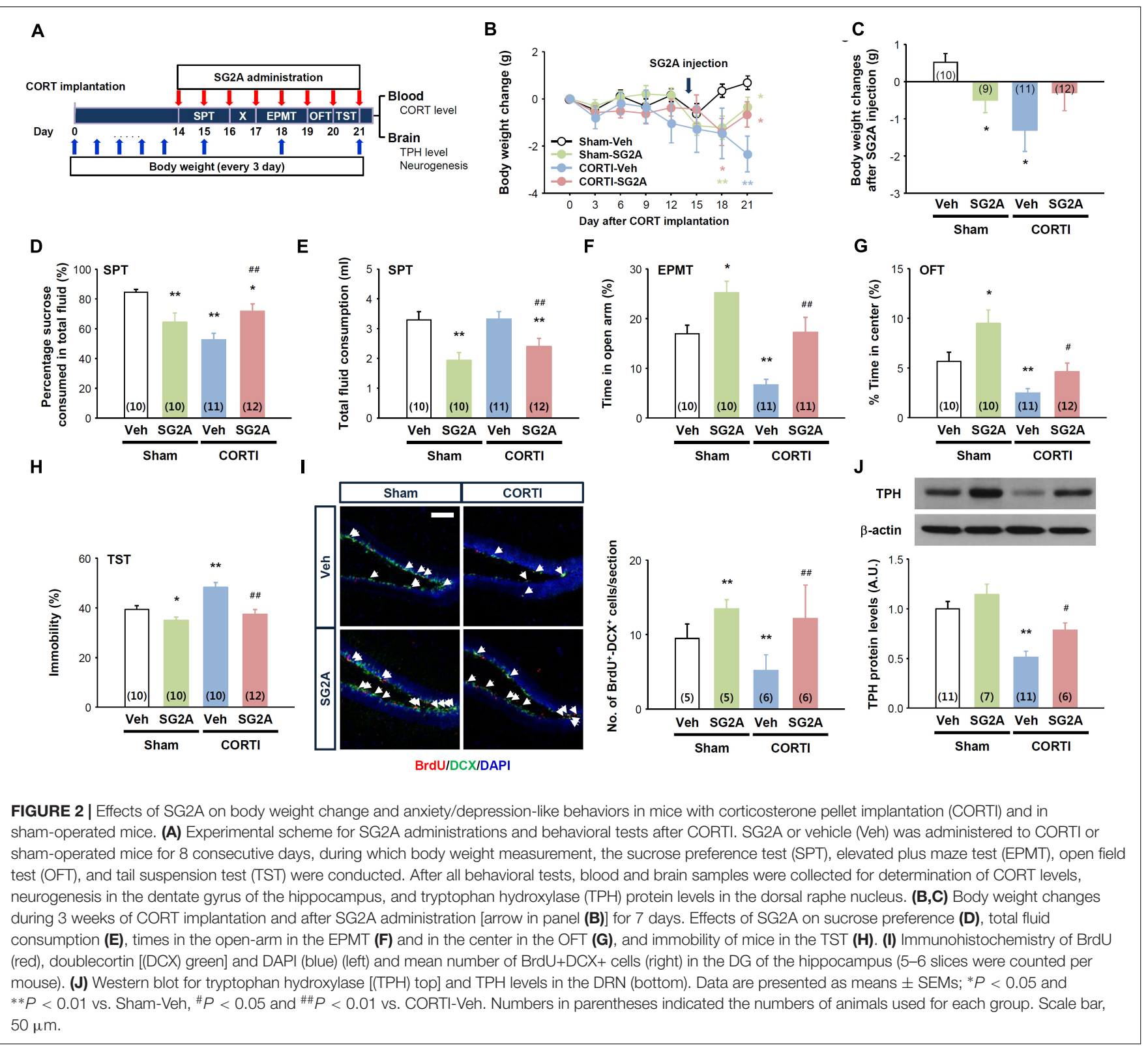

$4 \mathrm{~h}$ after SG2A administration on the last day, blood and brain samples were collected (Figure 2A).

High levels of blood corticosterone were maintained for 21 days in CORTI mice, regardless of SG2A administration (Supplementary Figure 1A), and the body weights of CORTI mice were significantly lower than the sham-operated/vehicletreated (Sham-Veh) mice (Figure 2B). Notably, the effect of SG2A on body weight differed between Sham and CORTI mice. The treatment of Sham mice with SG2A (Sham-SG2A) for 7 consecutive days led to a significant body weight loss, whereas the SG2A-treated CORTI mice partly recovered the body weights $(P=0.08$, CORTI-SG2A vs. CORTI-Veh) and did not differ from those of Sham-Veh mice (Figure 2C).

The results of the SPT suggest that CORTI induces anhedonia, a major symptom of depression, as no sucrose preference was observed in CORTI-Veh mice. SG2A administration significantly increased the sucrose preference rate in CORTI mice but induced a moderate but significant decrease in sucrose preference in Sham mice (Figure 2D). Notably, total fluid consumption did not differ between CORTI-Veh and Sham-Veh mice (Figure 2E), indicating that the decrease of sucrose preference in CORTIVeh mice is likely due to anhedonia. By contrast, the decrease of sucrose preference in Sham-SG2A and CORTI-SG2A mice accompanied decreased total fluid (Figure 2E) and sucrose (Supplementary Figure 1B) consumption.

In the EPMT, CORT-Veh mice appeared to be very anxious, as their time in the open-arms was significantly less than that of Sham-Veh mice. SG2A administration increased the time in the open-arms for both groups (Figure 2F). In the OFT, CORTIVeh mice spent less time in the center than Sham-Veh mice, 


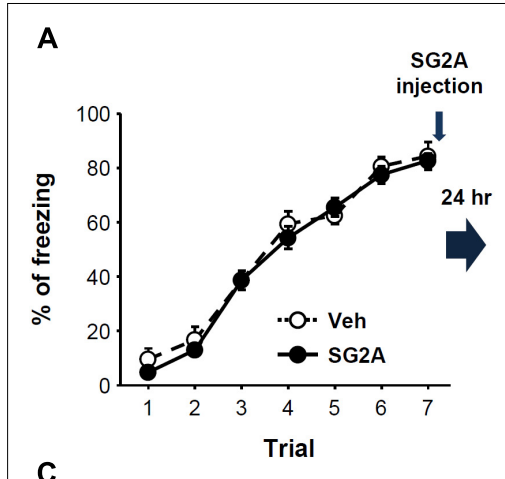

C

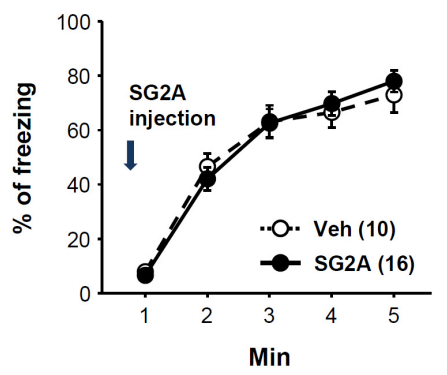

B

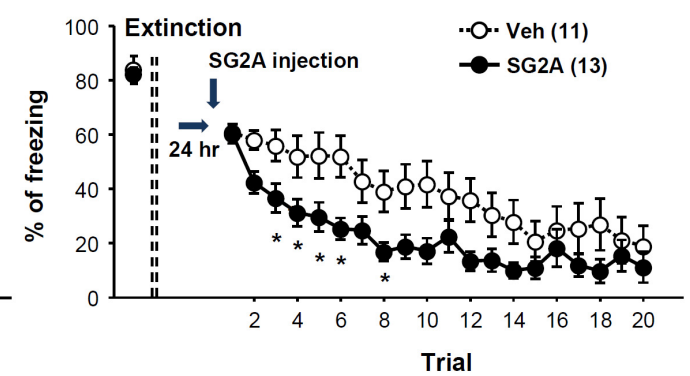

E
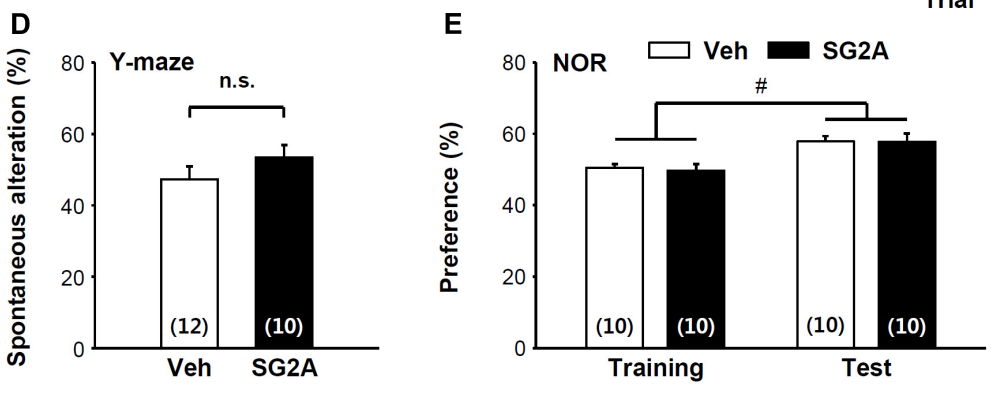

FIGURE 3 | Effect of SG2A on fear memory consolidation and extinction. (A) Decreased fear memory consolidation by SG2A. Fear memory acquisition curves during seven repeated tones and foot shock pairing (left). SG2A was administered immediately after acquisition, and contextual fear memory (middle) and auditory fear memory (right) were determined $24 \mathrm{~h}$ later. (B) Facilitated extinction of consolidated fear memory by SG2A. $24 \mathrm{~h}$ after memory acquisition, freezing behaviors were scored during 20 trials of a 30-s tone without foot shock in the distinct context in the presence or absence of SG2A. (C) Innate fear response is unaltered by SG2A. Freezing behaviors of mice exposed to TMT were scored. (D) Recognition memory by SG2A in the $Y$ maze. No significant difference (n.s.) was found between Veh- and SG2A-treated groups. (E) Novel object recognition test. Vehicle- and SG2A-treated mice similarly showed a preference for the novel object. Data are presented as means \pm SEMs; ${ }^{*} P<0.05$ and ${ }^{*} * P<0.01 \mathrm{vs}$. Veh, $\# P<0.05$ between training and test group. Numbers in parentheses indicated the numbers of animals used for each group.

indicating increased anxiety. This anxiogenic effect of CORTI was rescued by SG2A administration (Figure 2G). Total activities were not significantly different among the experimental groups (Supplementary Figure 1C).

In the TST, the immobility of CORTI-Veh mice was significantly higher than that of Sham-Veh mice, indicating an increased depression-like behavior. SG2A reduced the immobility of both groups (Figure $\mathbf{2 H}$ ).

The DG of the hippocampus is particularly sensitive to highly sustained corticosterone, showing altered neural death and adult neurogenesis. Furthermore, neurogenesis in the DG is highly associated with depression, as antidepressant drugs increase neurogenesis (Schmidt and Duman, 2007). Thus, proliferating neural progenitors in the DG were examined by counting $\mathrm{BrdU}^{+} \mathrm{DCX}^{+}$cells. The number of double-positive cells was significantly decreased in CORTI-Veh mice compared to that in Sham-Veh mice. SG2A treatment significantly augmented the number of $\mathrm{BrdU}^{+} \mathrm{DCX}^{+}$cells in both groups (Figure 2I).

Depression-like behavior reflects an underactivity of monoaminergic transmission. We examined protein levels of TPH, the rate-limiting enzyme for serotonin (5-HT) synthesis, as our data, presented below, suggested that SG2A activates 5-HT neurons in the dorsal raphe nucleus (DRN) but not dopaminergic neurons in substantia nigra (SN) and the ventral tegmental area (VTA). CORTI markedly lowered TPH levels compared to those of Sham-Veh mice. This decrease in TPH levels was rescued by SG2A (Figure 2J).

\section{Effects of SG2A on Fear Memory Consolidation and Extinction}

As SG2A exerted anxiolytic effects, we further determined the effects of SG2A on fear memory consolidation and extinction using the contextual and auditory-cued Pavlovian fear conditioning model. Seven trials of auditory cue with foot shock resulted in fear memory acquisition. Immediately after the last trial, SG2A or vehicle was administered into the LV of the mice (Figure 3A, left). The fear memory consolidation was determined $24 \mathrm{~h}$ later by placing the mice in the same chamber without auditory cues (context fear memory test) or in a different chamber with the auditory cues (auditory fear memory test). SG2A-administered mice spent significantly less time freezing than vehicle-treated mice in response to the context (Figure 3A, middle). Likewise, SG2A reduced the freezing rate responding to the auditory cue (Figure $\mathbf{3 A}$, right). Thus, SG2A administration just after fear memory acquisition reduced both contextual and auditory fear memory consolidation after $24 \mathrm{~h}$. Fear memory extinction was also examined at this time by determining the freezing rate in response to repeated auditory cues without foot-shock. SG2A or vehicle was administered 2-3 h 


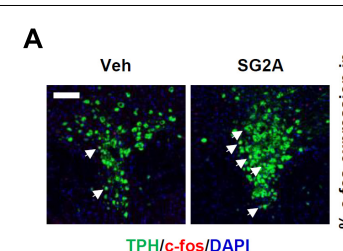

D

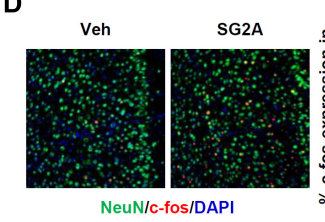

G

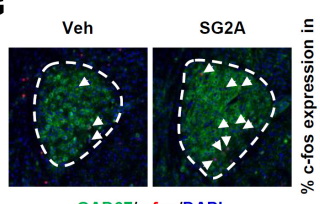

J

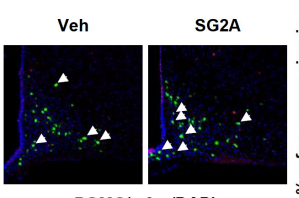

POMC/c-fos/DAP
B

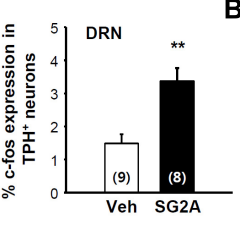

E

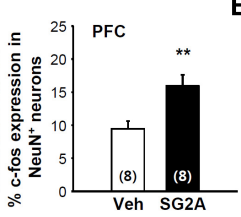

E
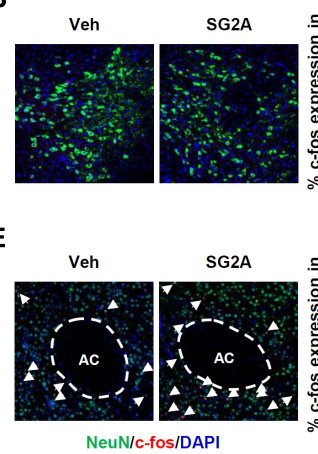
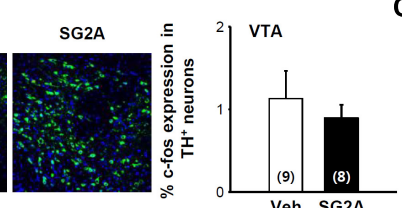

Veh SG2A

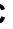

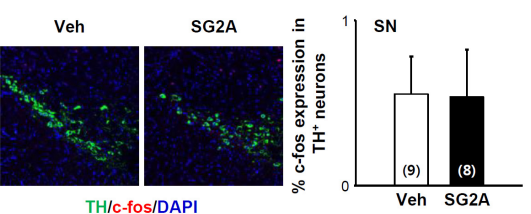

$\mathbf{F}$
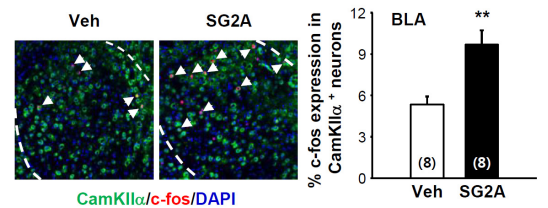

$\mathrm{H}$
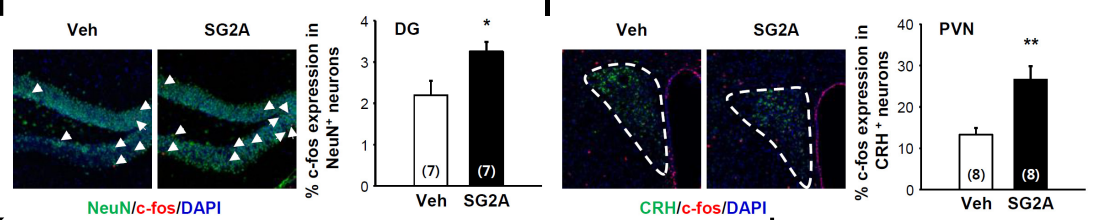

K
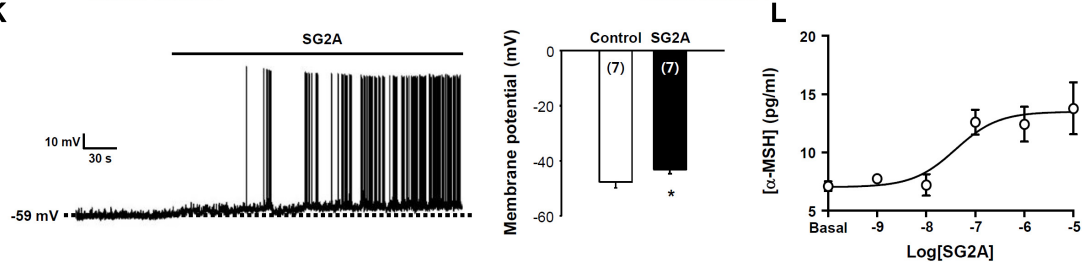

FIGURE 4 | Neurons responding to SG2A. Immunohistochemistry for c-fos ${ }^{+}$(red) neurons in mice administered SG2A or vehicle (Veh) for 1-2 h. The Percentage of cells double immunopositive for c-fos and TPH (serotonergic neurons) in the DRN (A), TH (dopaminergic neurons) in the VTA (B) and SN (C), NeuN in PFC (D) and NAc (E), CamKIl $\alpha$ in BLA (F), GAD67 in CeA (G), NeuN in the DG of the hippocampus (H), CRH in PVN (I), and POMC in ARC (J) were counted (3-4 slices were counted per mouse). (K) Depolarization of POMC neuron membrane potential in response to applications of SG2A (left); summary of acute effects of SG2A on the membrane potentials of responsive POMC neurons (right). (L) $\alpha$-MSH secretion in cultured POMC neurons in response to SG2A. Data are presented as means \pm SEMs; ${ }^{*} P<0.05$ and ${ }^{*} P<0.01$ vs. Veh. Numbers in parentheses indicate the numbers of animals used for each group. Scale bar, 50 m.

before the first auditory cue. Vehicle-treated mice showed a gradual decrease in freezing rate as the number of trials increased, and SG2A accelerated this decrease beginning at the second trial [treatment effect: $F(1,22)=5.192, P<0.05$; interaction: $F(1,22)=1.773, P<0.05]$ (Figure 3B). These results suggest that SG2A facilitates the extinction of fear memories. Whether these effects were due to a decreased response to fear-causing environments (unconditioned fear response) or to impaired recognition memory was not clear. However, SG2A did not alter unconditioned fear responses, as freezing behaviors of mice exposed to TMT, a synthetic fox (predator) feces odor, did not differ (Figure 3C). The results from the Y-maze test suggest the SG2A does not alter spatial recognition memory, as there was no difference between vehicle- and SG2A-injected mice (Figure 3D). Furthermore, SG2A did not influence responding in the NOR test (Figure 3E). Thus the effects of SG2A on fear memory were not attributable to a decreased response to a fear environment or an impairment of recognition memory.

\section{Neuronal Activation by SG2A}

To determine the possible neural circuits for SG2A action, mRNA expression of SPX/GAL and GAL receptors were determined in the various brain regions using in situ hybridization assay and quantitative RT-PCR. SPX mRNA were strongly detected in the medial habenula $(\mathrm{mHb})$ and suprachiasmatic nucleus (SCN) of the hypothalmus. Interestingly, $\mathrm{GAL}_{2}$ receptor mRNA levels were high in the hypothalamus, $\mathrm{Hb}$, ventral midbrain (VMB), and DRN which are potential target regions of SPX action. In contrast, mRNA expressions of GAL, GAL $\mathrm{G}_{1}$ receptor, and $\mathrm{GAL}_{3}$ receptor were mainly restricted in the hypothalamus (Supplementary Figure 2). To identify the neural circuits influenced by $\mathrm{GAL}_{2}$ receptor activation, the numbers of $\mathrm{c}$-fos ${ }^{+}$ neurons in various brain regions were determined at $1-2 \mathrm{~h}$ after a single i.c.v. administration of SG2A, which is sufficient to induce anxiolytic-, antidepressant-, and anorexic-like effects in normal and ob/ob mice (Supplementary Figure 3). We found that SG2A significantly increased the number of $\mathrm{c}$-fos ${ }^{+} \mathrm{TPH}^{+}$ 5 -HT neurons in the DRN (Figure 4A) but not the number of $\mathrm{c}$-fos and $\mathrm{TH}$ double-positive dopaminergic neurons in the VTA (Figure 4B) and SN (Figure 4C). SG2A administration also induced $\mathrm{c}$-fos in the prefrontal cortex (PFC) (Figure 4D) and the nucleus accumbens (NAc) (Figure 4E), regions implicated in the pathophysiology of depression and innervated by 5-HT neurons from the DRN (Challis and Berton, 2015). c-fos expression was also increased in the basolateral (BLA) and central amygdala (CeA). In particular, the number of neurons immunoreactive for both $\mathrm{c}$-fos and CamKII $\alpha$ (Figure 4F) or GAD67 (Figure 4G) was higher in SG2A-injected mice than in vehicle-treated mice. In the 
A

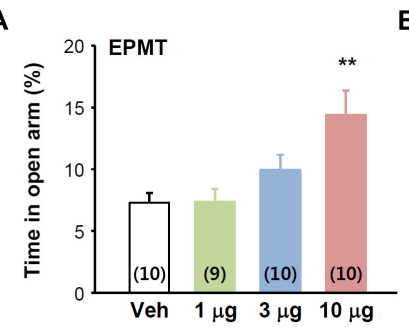

E

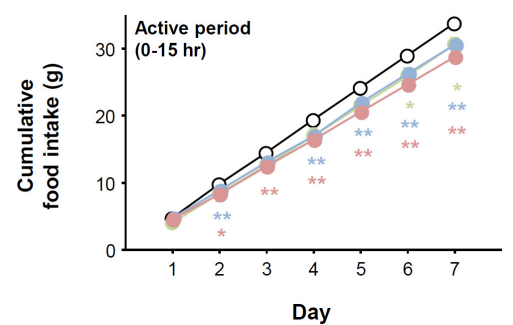

B

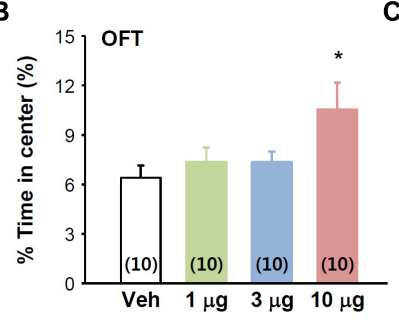

C

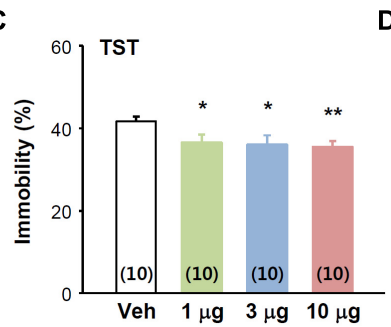

D

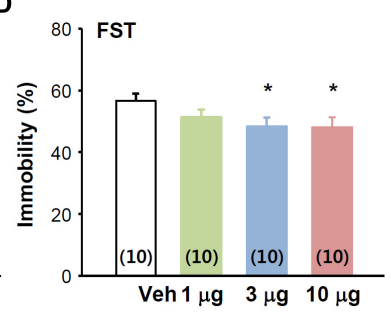

F
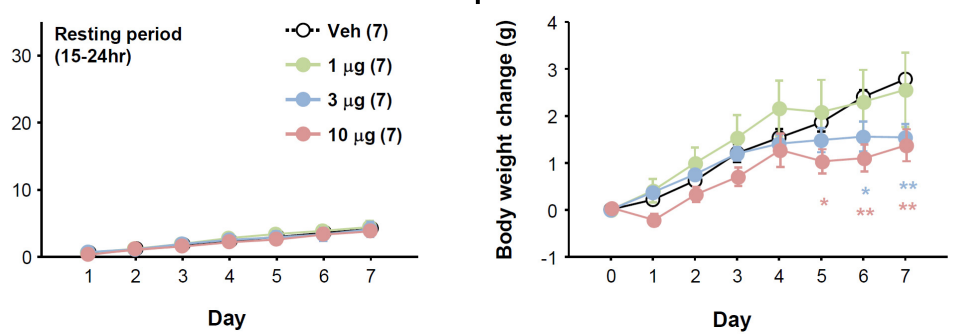

FIGURE 5 | Effects of intranasally administered SG2A on anxiety, depression-like, and feeding behaviors. Normal mice received various concentrations of SG2A (1, 3, and $10 \mu \mathrm{g} /$ mouse). Anxiolytic effect of SG2A in the EPMT (A) and OFT (B). Antidepressant-like effect of SG2A in the TST (C) and FST (D). Cumulative changes in food intake (E) and body weight (F) during 7 consecutive administrations of SG2A. Data are presented as means $\pm \mathrm{SEMs} ;{ }^{*} P<0.05$ and ${ }^{* *} P<0.01$ vs. Veh.

Numbers in parentheses indicate the numbers of animals used for each group.

hippocampus, SG2A increased the number of $\mathrm{c}-$ fos $^{+}$neurons in DG (Figure 4H) but not in CA1, CA2, or CA3 (Supplementary Figure 4). The activation of neurons in the amygdala and DG is implicated in regulating anxiety and fear memory (SierraMercado et al., 2011). Increased c-fos immunoreactivity was also observed in CRH neurons in the paraventricular nucleus (PVN) (Figure 4I) and POMC neurons in the arcuate nucleus (ARC) (Figure 4J). However, other brain regions, such as $\mathrm{Hb}$, lateral hypothalamus, and SCN, did not show c-fos induction after SG2A administration (Supplementary Figure 4).

As POMC neurons in the ARC are particularly important for regulating body weight via appetite and energy expenditure (Schwartz et al., 2000), we further examined whether SG2A can directly activate these neurons ex vivo and in vitro. POMC neurons in hypothalamic slices obtained from POMC-hrGFP transgenic mice can be identified by their green fluorescence (Sohn et al., 2011). As shown in Figure 4K, bath application of SG2A depolarized the membrane potentials of $7 / 18$ (38.9\%) POMC neurons by $4.6 \pm 0.6 \mathrm{mV}$ (from $-47.7 \pm 2.1$ to $-43.1 \pm 1.5 \mathrm{mV}$, $P<0.05)$. The other 11 cells remained unresponsive to SG2A (from $-46.8 \pm 2.2$ to $-46.6 \pm 2.2 \mathrm{mV}, P>0.05$ ). These data demonstrate that $\mathrm{GAL}_{2}$ receptor stimulation excites POMC neurons. In vitro, cultured POMC neurons exhibited dose-dependent SG2A-induced secretion of $\alpha-\mathrm{MSH}$, a major anorexic neuropeptide (Figure 4L; Yang and Tao, 2017).

\section{Nasal Application of SG2A}

The effects of SG2A in these animal models suggest it has therapeutic potential. Thus, we examined whether SG2A can be delivered to the CNS by i.n. administration, a non-invasive route for enabling efficient crossing of the blood-brain barrier (Kageyama et al., 2016). Behavioral tests were performed 2-3 h after SG2A i.n. administration. Similar to that observed with i.c.v. administration, mice receiving i.n. SG2A spent more time in the open-arms in the EPMT (Figure 5A) and open field in OFT (Figure 5B) without significant changes in total movement (Supplementary Figure $\mathbf{5 A}$ ) and significantly less immobility in the TST (Figure 5C) and FST (Figure 5D), suggesting that i.n. SG2A reduced anxiety- and depression-like behaviors. When administered for 7 days, i.n. SG2A significantly decreased cumulative food intake and body weight in a dose-dependent manner. SG2A decreased food intake during the active period when the mice mainly consume the chow, but not during the resting period (Figure 5E). These decreases corresponded to continual decreases in body weight, with a significant difference versus the vehicle-treated group observed beginning day 5 after SG2A administration for the $10 \mu \mathrm{g}$-treated group and day 6 for the $3 \mu \mathrm{g}$-treated group (Figure 5F).

Successful delivery of SG2A to the brain via the i.n. route was confirmed by $\mathrm{c}$-fos induction in the brain regions that responded to i.c.v. administration. Specifically, i.n. administration of SG2A increased the numbers of $\mathrm{c}-\mathrm{fos}^{+} \mathrm{TPH}^{+}$ 5-HT neurons in the DRN, c-fos ${ }^{+} \mathrm{NeuN}^{+}$neurons in the PFC, NAc, and DG, c-fos ${ }^{+}$CamKII $\alpha^{+}$cells in the BLA, and $\mathrm{c}^{-} \mathrm{fos}^{+} \mathrm{GAD}^{+} 7^{+}$cells in the CeA (Supplementary Figure 5). Antidepressive and anxiolytic effects of SG2A i.n. administration were also examined in CORTI mice. Nasal delivery of SG2A increased the time in the open-arm in the EPMT, increased time in the center in OFT, and decreased the time of immobility in the TST without any 
differences in total activities (Supplementary Figure 6). Altogether, these results demonstrate that SG2A can be delivered to the CNS via an i.n. route to sufficiently evoke neurochemical and behavioral effects similar to those observed after i.c.v. administration.

\section{DISCUSSION}

Considering the cross-reactivity of SPX and GAL toward GAL receptors and the overall complexity of the GAL receptor subtype-specific signaling pathways (Kim et al., 2014; Yun et al., 2014), the development of subtype-selective GAL receptor agonists may help to elucidate GAL receptor subtype-specific physiological functions (Reyes-Alcaraz et al., 2016). Many different types of GAL receptor-targeting agonists/antagonists have been generated by using a variety of fragments from the GAL peptide. However, these agents maintained their substantial affinity toward other GAL receptor subtypes at high concentrations (Webling et al., 2012). Recently, we developed SG2A, an SPX-based $\mathrm{GAL}_{2}$ receptor agonist that lacks activity toward $\mathrm{GAL}_{1}$ receptor and $\mathrm{GAL}_{3}$ receptor (ReyesAlcaraz et al., 2016). The pharmacological relevance of SG2A is underscored by its biased agonism toward G-proteindependent signaling over $\beta$-arrestin-mediated signaling, which is similar to SPX but different from GAL with unbiased activation (Reyes-Alcaraz et al., 2018). Probably due to $\beta$-arrestin-mediated signaling, classical agonists often result in contradictory results, such as with GAL for depressionlike behaviors ( $\mathrm{Lu}$ et al., 2005; Kuteeva et al., 2008) and the dichotomous action/dose-dependent inaction of galaninlike peptide in regulating food intake (Lawrence et al., 2002; Kageyama et al., 2016). By contrast, SG2A shows dosedependent and consistent effects on mood and appetite behaviors during repetitive administrations. Thus, compared to the GALbased agonist, the SPX-based agonist may have multiple pharmacological benefits, exerting its action on both mood and appetite/body weight.

SG2A administration (i.c.v./i.n.) produced rapid anxiolyticand antidepressant-like behavioral effects. Thus, time required for onset of effects is different from selective serotonin reuptake inhibitors (SSRIs) that show a late onset of antidepressive effects. Nevertheless, the effect of SG2A is likely mediated by activation of 5 -HT neurons in the DRN as shown in Figure 4A. It is of interesting to note that SPX mRNA is highly expressed in the $\mathrm{mHb}$. Projection from $\mathrm{mHb}$ to interpeduncular nucleus (IPN) is connected to the serotonergic raphe nucleus which is implicated in the regulation of mood such as depression and anxiety (Yamaguchi et al., 2013). In addition, the DRN is known to express $\mathrm{GAL}_{2}$ receptor which then promote TPH transcription (Xu et al., 1998; Lu et al., 2005; Mazarati et al., 2005). Indeed, our study showed that repetitive SG2A administration significantly increased $\mathrm{TPH}$ protein levels in the DRN of CORTI mice. Interestingly, chronic treatment of fluoxetine, an SSRI, increases $\mathrm{GAL}_{2}$ receptor binding sites on the DRN (Lu et al., 2005). Thus, both SG2A and SSRI are involved in direct or indirect activation of $\mathrm{GAL}_{2}$ receptor for increased activities of 5-HT neurons in the DRN. In addition, SG2A, similar to SSRIs, increases neurogenesis in the DG of the hippocampus. The hippocampal neurogenesis is decreased by inescapable stress events resulting in a state of behavioral despair and this effect can be reversed by fluoxetine (Malberg and Duman, 2003). Disruption of fluoxetine-induced neurogenesis suppresses behavioral responses to antidepressants, therefore the increased neurogenesis is likely an essential process for antidepressive behavioral effects (Santarelli et al., 2003). Thus, increase in $\mathrm{c}$-fos ${ }^{+} \mathrm{TPH}^{+}$5-HT neurons by SG2A and similarity of histochemical changes in the brain between SSRI and SG2A may suggest that antidepressive and anxiolytic effects of SG2A in CORTI mice are mainly mediated by activation of 5-HT neurons in the DRN.

One of the unexpected side effects of SSRIs is body weight changes (Levine et al., 1987; Uguz et al., 2015). A recent study showed that long-term treatment of escitalopram, an effective SSRI antidepressant that is associated with significant weight gain (Uguz et al., 2015), led to the downregulation of SPX expression in the rat hypothalamus. This suggests that the increased body mass by SSRI may be, at least in part, due to a decreased anorexic action of SPX in the hypothalamus (Palasz et al., 2016). Both POMC and $\mathrm{CRH}$ neurons in the hypothalamus are pivotal neuronal populations involved in appetite/body weight regulation (Schwartz et al., 2000). In goldfish, i.c.v. administration of SPX inhibited food intake accompanying increased mRNA levels of POMC and CRH (Wong et al., 2013). Our results also showed that $\mathrm{SG} 2 \mathrm{~A}$ reduced food consumption with induced $\mathrm{c}$-fos expression in POMC- and CRH-positive neurons. As SG2A reduced body weight in normal and obese mice, SG2A treatment can circumvent SSRI-induced increase in body weight by direct activation of anorexic neuronal population in the hypothalamus. POMC and $\mathrm{CRH}$ neurons in the hypothalamus sense and integrate inputs not only from peripheral tissues (Schwartz et al., 2000) but also central inputs from brain areas involved in stress/mood/hedonic/reward control (Adam and Epel, 2007; Carroll et al., 2012). Moreover, hedonic or reward-based control can override homeostatic pathway for eating behaviors and body weight controls. In CORTI mice that already lost body weight, SG2A increased their body weight, likely via relieving anhedonia, anxiety, and depression. Thus, it seems likely that the antidepressive effects of SG2A overwhelms the anorexic effects of SG2A in CORTI mice. Together, SPX and $\mathrm{GAL}_{2}$ receptor are likely connected to the key regulatory system controlling or linking appetite and mood behaviors, allowing optimal treatment for comorbid mood disorders and abnormal body weight.

Post-traumatic stress disorder (PTSD) is a severe anxiety disorder that involves an explicit conditioning episode (Dohrenwend et al., 2006; de Vries and Olff, 2009). Furthermore, PTSD and depression are commonly co-occurring mental disorders which reinforces each other. PTSD is frequently conceptualized as a memory disorder within a Pavlovian fear conditioning and extinction framework (Rubin et al., 2008; 
Shin and Handwerger, 2009). This study also showed that SG2A contributes to fear memory consolidation and extinction in a PTSD animal model. Specifically, SG2A decreased both hippocampus-dependent contextual and amygdala-dependent auditory fear responses and facilitated fear extinction after fear memory acquisition. Accordingly, SG2A administration activated neurons in the amygdala, PFC, and DG which are major components of the neural circuits that regulate anxiety and fear memory (Sierra-Mercado et al., 2011). Although there are reports of impaired cognition induced by GAL (Rustay et al., 2005), SG2A did not induce memory deficits in the Y-maze and NOR tests in this study.

A major challenge for treatments involving peptide drugs is bypassing the blood-brain barrier. Over the last several decades, diverse formulations and devices have been developed to transport the drugs from the nose directly to the brain, showing promise for therapeutic efficacy based on animal models and clinical trials in humans (Craft et al., 2012; Striepens et al., 2013; Kageyama et al., 2016). Notably, i.n. administrations of GLP2, oxytocin, or other peptides produce antidepressive effects or improve social behavior, thereby attracting attention to the development of peptide drugs for the treatment of neuropsychiatric diseases (SasakiHamada et al., 2017). The results of this study showed that SG2A can be successfully delivered to the CNS via the i.n. route, producing results comparable to those obtained with i.c.v. delivery.

\section{CONCLUSION}

In conclusion, SG2A exerts a rapid onset of effects toward relieving anxiety-, depression-like, and feeding behaviors and suppressing fear memory. These effects were maintained during repetitive SG2A administrations delivered either i.c.v./i.n. SG2A has potential for the clinical application to treat mood disorders and/or abnormal appetite/body weight.

\section{REFERENCES}

Adam, T. C., and Epel, E. S. (2007). Stress, eating and the reward system. Physiol. Behav. 91, 449-458. doi: 10.1016/j.physbeh.2007.04.011

American Psychiatric Association (2013). Diagnostic and Statistical Manual of Mental Disorders, 5th Edn. Arlington, VA: American Psychiatric Publishing.

Bailey, K. R., Pavlova, M. N., Rohde, A. D., Hohmann, J. G., and Crawley, J. N. (2007). Galanin receptor subtype 2 (GalR2) null mutant mice display an anxiogenic-like phenotype specific to the elevated plus-maze. Pharmacol. Biochem. Behav. 86, 8-20. doi: 10.1016/j.pbb.2006.11.024

Baranowska, B., Wasilewska-Dziubinska, E., Radzikowska, M., Plonowski, A., and Roguski, K. (1997). Neuropeptide Y, galanin, and leptin release in obese women and in women with anorexia nervosa. Metabolism 46, 1384-1389. doi: 10.1016/ s0026-0495(97)90136-0

Carey, M., Small, H., Yoong, S. L., Boyes, A., Bisquera, A., and Sanson-Fisher, R. (2014). Prevalence of comorbid depression and obesity in general practice: a cross-sectional survey. Br. J. Gen. Pract. 64, e122-e127. doi: 10.3399/ bjgp14X677482

Carroll, B. J., Iranmanesh, A., Keenan, D. M., Cassidy, F., Wilson, W. H., and Veldhuis, J. D. (2012). Pathophysiology of hypercortisolism in depression:

\section{DATA AVAILABILITY}

All datasets generated for this study are included in the manuscript and/or the Supplementary Files.

\section{ETHICS STATEMENT}

All animal procedures were approved by the Institutional Animal Care and Use Committee of Korea University (KOREA2016-0050-C2) and Korea Advanced Institute of Science and Technology (KA2014-02).

\section{AUTHOR CONTRIBUTIONS}

SY, AR-A, B-JH, J-WS, D-HK, GS, HK, S-GK, DK, and BK designed the research. SY, AR-A, Y-NL, HY, and JC performed the research. SY and AR-A analyzed the data. J-IH and JS wrote the manuscript. All authors read and approved the final manuscript.

\section{FUNDING}

This work was supported by grants from the Research Program of the National Research Foundation of Korea (NRF2015M3A9E7029172) funded by the Ministry of Science, ICT, and Future Planning. Neuracle Science Co., Ltd., holds a Korean patent (10-1885238 and 10-1885241), United States (15/771,078), EPO (16871028.3), Australian (2016361783), Brazilian (11 2018008315 1), Canada (3003262), China (201680062527.4), and Japan (2018-523789) patents.

\section{SUPPLEMENTARY MATERIAL}

The Supplementary Material for this article can be found online at: https://www.frontiersin.org/articles/10.3389/fnins. 2019.00391/full\#supplementary-material

pituitary and adrenal responses to low glucocorticoid feedback. Acta. Psychiatr. Scand. 125, 478-491. doi: 10.1111/j.1600-0447.2011.01821.x

Challis, C., and Berton, O. (2015). Top-down control of serotonin systems by the prefrontal cortex: a path toward restored socioemotional function in depression. ACS Chem. Neurosci. 6, 1040-1054. doi: 10.1021/acschemneuro. 5 b00007

Craft, S., Baker, L. D., Montine, T. J., Minoshima, S., Watson, G. S., Claxton, A., et al. (2012). Intranasal insulin therapy for Alzheimer disease and amnestic mild cognitive impairment: a pilot clinical trial. Arch. Neurol. 69, 29-38. doi: 10.1001/archneurol.2011.233

de Vries, G. J., and Olff, M. (2009). The lifetime prevalence of traumatic events and posttraumatic stress disorder in the Netherlands. J. Trauma Stress 22, 259-267. doi: 10.1002/jts.20429

Demuyser, T., Bentea, E., Deneyer, L., Albertini, G., Massie, A., and Smolders, I. (2016). Disruption of the HPA-axis through corticosterone-release pellets induces robust depressive-like behavior and reduced BDNF levels in mice. Neurosci. Lett. 626, 119-125. doi: 10.1016/j.neulet.2016. 05.026

Dohrenwend, B. P., Turner, J. B., Turse, N. A., Adams, B. G., Koenen, K. C., and Marshall, R. (2006). The psychological risks of Vietnam for U.S. veterans: a 
revisit with new data and methods. Science 313, 979-982. doi: 10.1126/science. 1128944

Furlong, M., and Seong, J. Y. (2017). Evolutionary and comparative genomics to drive rational drug design, with particular focus on neuropeptide seventransmembrane receptors. Biomol. Ther. 25, 57-68. doi: 10.4062/biomolther. 2016.199

Hausdorff, W. P., Caron, M. G., and Lefkowitz, R. J. (1990). Turning off the signal: desensitization of beta-adrenergic receptor function. FASEB J. 4, 2881-2889. doi: 10.1096/fasebj.4.11.2165947

Kageyama, H., Shiba, K., Hirako, S., Wada, N., Yamanaka, S., Nogi, Y., et al. (2016). Anti-obesity effect of intranasal administration of galaninlike peptide (GALP) in obese mice. Sci. Rep. 6:28200. doi: 10.1038/srep2 8200

Karatayev, O., Baylan, J., and Leibowitz, S. F. (2009). Increased intake of ethanol and dietary fat in galanin overexpressing mice. Alcohol 43, 571-580. doi: 10 . 1016/j.alcohol.2009.09.025

Kim, D. K., Yun, S., Son, G. H., Hwang, J. I., Park, C. R., Kim, J. I., et al. (2014). Coevolution of the spexin/galanin/kisspeptin family: spexin activates galanin receptor type II and III. Endocrinology 155, 1864-1873. doi: 10.1210/en.20132106

Kloiber, S., Ising, M., Reppermund, S., Horstmann, S., Dose, T., Majer, M., et al. (2007). Overweight and obesity affect treatment response in major depression. Biol. Psychiatry 62, 321-326. doi: 10.1016/j.biopsych.2006. 10.001

Kuteeva, E., Hokfelt, T., Wardi, T., and Ogren, S. O. (2008). Galanin, galanin receptor subtypes and depression-like behaviour. Cell. Mol. Life. Sci. 65, 18541863. doi: 10.1007/s00018-008-8160-9

Lawrence, C. B., Baudoin, F. M., and Luckman, S. M. (2002). Centrally administered galanin-like peptide modifies food intake in the rat: a comparison with galanin. J. Neuroendocrinol. 14, 853-860. doi: 10.1046/j.1365-2826.2002. 00846.x

Levine, L. R., Rosenblatt, S., and Bosomworth, J. (1987). Use of a serotonin reuptake inhibitor, fluoxetine, in the treatment of obesity. Int. J. Obes. 11(Suppl. 3), 185-190.

Lu, X., Barr, A. M., Kinney, J. W., Sanna, P., Conti, B., Behrens, M. M., et al. (2005). A role for galanin in antidepressant actions with a focus on the dorsal raphe nucleus. Proc. Natl. Acad. Sci. U.S.A. 102, 874-879. doi: 10.1073/pnas. 0408891102

Malberg, J. E., and Duman, R. S. (2003). Cell proliferation in adult hippocampus is decreased by inescapable stress: reversal by fluoxetine treatment. Neuropsychopharmacology 28, 1562-1571. doi: 10.1038/sj.npp.1300234

Marijnissen, R. M., Bus, B. A., Holewijn, S., Franke, B., Purandare, N., De Graaf, J., et al. (2011). Depressive symptom clusters are differentially associated with general and visceral obesity. J. Am. Geriatr. Soc. 59, 67-72. doi: 10.1111/j.15325415.2010.03228.x

Mazarati, A. M., Baldwin, R. A., Shinmei, S., and Sankar, R. (2005). In vivo interaction between serotonin and galanin receptors types 1 and 2 in the dorsal raphe: implication for limbic seizures. J. Neurochem. 95, 1495-1503. doi: 10.1111/j.1471-4159.2005.03498.x

Palasz, A., Suszka-Switek, A., Filipczyk, L., Bogus, K., Rojczyk, E., Worthington, J., et al. (2016). Escitalopram affects spexin expression in the rat hypothalamus, hippocampus and striatum. Pharmacol. Rep. 68, 1326-1331. doi: 10.1016/j. pharep.2016.09.002

Reyes-Alcaraz, A., Lee, Y. N., Son, G. H., Kim, N. H., Kim, D. K., Yun, S., et al. (2016). Development of spexin-based human galanin receptor type II-specific agonists with increased stability in serum and anxiolytic effect in mice. Sci. Rep. 6:21453. doi: 10.1038/srep2 1453

Reyes-Alcaraz, A., Lee, Y. N., Yun, S., Hwang, J. I., and Seong, J. Y. (2018). Conformational signatures in beta-arrestin2 reveal natural biased agonism at a G-protein-coupled receptor. Commun. Biol. 1:128. doi: 10.1038/s42003-0180134-3

Rubin, D. C., Berntsen, D., and Bohni, M. K. (2008). A memory-based model of posttraumatic stress disorder: evaluating basic assumptions underlying the PTSD diagnosis. Psychol. Rev. 115, 985-1011. doi: 10.1037/a001 3397

Rustay, N. R., Wrenn, C. C., Kinney, J. W., Holmes, A., Bailey, K. R., Sullivan, T. L., et al. (2005). Galanin impairs performance on learning and memory tasks: findings from galanin transgenic and GAL-R1 knockout mice. Neuropeptides 39, 239-243. doi: 10.1016/j.npep.2004. 12.026

Santarelli, L., Saxe, M., Gross, C., Surget, A., Battaglia, F., Dulawa, S., et al. (2003). Requirement of hippocampal neurogenesis for the behavioral effects of antidepressants. Science 301, 805-809. doi: 10.1126/science.108 3328

Sasaki-Hamada, S., Nakamura, R., Nakao, Y., Akimoto, T., Sanai, E., Nagai, M., et al. (2017). Antidepressant-like effects exerted by the intranasal administration of a glucagon-like peptide- 2 derivative containing cell-penetrating peptides and a penetration-accelerating sequence in mice. Peptides 87, 64-70. doi: 10.1016/j. peptides.2016.11.013

Schmidt, H. D., and Duman, R. S. (2007). The role of neurotrophic factors in adult hippocampal neurogenesis, antidepressant treatments and animal models of depressive-like behavior. Behav. Pharmacol. 18, 391-418. doi: 10.1097/FBP. 0b013e3282ee $2 \mathrm{aa} 8$

Schwartz, M. W., Woods, S. C., Porte, D. Jr., Seeley, R. J., and Baskin, D. G. (2000). Central nervous system control of food intake. Nature 404, 661-671. doi: $10.1038 / 35007534$

Sharma, S., Fernandes, M. F., and Fulton, S. (2013). Adaptations in brain reward circuitry underlie palatable food cravings and anxiety induced by high-fat diet withdrawal. Int. J. Obes. 37, 1183-1191. doi: 10.1038/ijo.20 12.197

Shin, L. M., and Handwerger, K. (2009). Is posttraumatic stress disorder a stressinduced fear circuitry disorder? J. Trauma Stress 22, 409-415. doi: 10.1002/jts. 20442

Sierra-Mercado, D., Padilla-Coreano, N., and Quirk, G. J. (2011). Dissociable roles of prelimbic and infralimbic cortices, ventral hippocampus, and basolateral amygdala in the expression and extinction of conditioned fear. Neuropsychopharmacology 36, 529-538. doi: 10.1038/npp.20 10.184

Simon, G. E., Von Korff, M., Saunders, K., Miglioretti, D. L., Crane, P. K., Van Belle, G., et al. (2006). Association between obesity and psychiatric disorders in the US adult population. Arch. Gen. Psychiatry 63, 824-830. doi: 10.1001/archpsyc.63. 7.824

Sohn, J. W., Xu, Y., Jones, J. E., Wickman, K., Williams, K. W., and Elmquist, J. K. (2011). Serotonin 2C receptor activates a distinct population of arcuate pro-opiomelanocortin neurons via TRPC channels. Neuron 71, 488-497. doi: 10.1016/j.neuron.2011.06.012

Striepens, N., Kendrick, K. M., Hanking, V., Landgraf, R., Wullner, U., Maier, W., et al. (2013). Elevated cerebrospinal fluid and blood concentrations of oxytocin following its intranasal administration in humans. Sci. Rep. 3:3440. doi: $10.1038 /$ srep03440

Swanson, C. J., Blackburn, T. P., Zhang, X., Zheng, K., Xu, Z. Q., Hokfelt, T., et al. (2005). Anxiolytic- and antidepressant-like profiles of the galanin-3 receptor (Gal3) antagonists SNAP 37889 and SNAP 398299. Proc. Natl. Acad. Sci. U.S.A. 102, 17489-17494. doi: 10.1073/pnas.050897 0102

Uguz, F., Sahingoz, M., Gungor, B., Aksoy, F., and Askin, R. (2015). Weight gain and associated factors in patients using newer antidepressant drugs. Gen. Hosp. Psychiatry 37, 46-48. doi: 10.1016/j.genhosppsych.2014.10.011

Walewski, J. L., Ge, F., Lobdell, H. T., Levin, N., Schwartz, G. J., Vasselli, J. R., et al. (2014). Spexin is a novel human peptide that reduces adipocyte uptake of long chain fatty acids and causes weight loss in rodents with diet-induced obesity. Obesity 22, 1643-1652. doi: 10.1002/oby. 20725

Webling, K. E., Runesson, J., Bartfai, T., and Langel, U. (2012). Galanin receptors and ligands. Front. Endocrinol. 3:146. doi: 10.3389/fendo.2012.00146

Wong, M. K., Sze, K. H., Chen, T., Cho, C. K., Law, H. C., Chu, I. K., et al. (2013). Goldfish spexin: solution structure and novel function as a satiety factor in feeding control. Am. J. Physiol. Endocrinol. Metab. 305, E348-E366. doi: 10.1152/ajpendo.00141. 2013

Xu, Z. Q., Zhang, X., Pieribone, V. A., Grillner, S., and Hokfelt, T. (1998). Galanin5-hydroxytryptamine interactions: electrophysiological, immunohistochemical and in situ hybridization studies on rat dorsal raphe neurons with a note on galanin R1 and R2 receptors. Neuroscience 87, 79-94. doi: 10.1016/s03064522(98)00151-1 
Yamaguchi, T., Danjo, T., Pastan, I., Hikida, T., and Nakanishi, S. (2013). Distinct roles of segregated transmission of the septo-habenular pathway in anxiety and fear. Neuron 78, 537-544. doi: 10.1016/j.neuron.2013. 02.035

Yang, L. K., and Tao, Y. X. (2017). Biased signaling at neural melanocortin receptors in regulation of energy homeostasis. Biochim. Biophys. Acta. 1863, 2486-2495. doi: 10.1016/j.bbadis.2017.04.010

Yun, S., Furlong, M., Sim, M., Cho, M., Park, S., Cho, E. B., et al. (2015). Prevertebrate local gene duplication facilitated expansion of the neuropeptide GPCR superfamily. Mol. Biol. Evol. 32, 2803-2817. doi: 10.1093/molbev/ msv179

Yun, S., Kim, D. K., Furlong, M., Hwang, J. I., Vaudry, H., and Seong, J. Y. (2014). Does kisspeptin belong to the proposed RF-amide peptide family? Front. Endocrinol. 5:134. doi: 10.3389/fendo.2014. 00134
Conflict of Interest Statement: SY, AR-A, Y-NL, HY, GS, J-IH, and JS are shareholders. S-GK and DK are employees and shareholders. BK is a shareholder and the CEO of Neuracle Science Co., Ltd.

The remaining authors declare that the research was conducted in the absence of any commercial or financial relationships that could be construed as a potential conflict of interest.

Copyright (c) 2019 Yun, Reyes-Alcaraz, Lee, Yong, Choi, Ham, Sohn, Kim, Son, Kim, Kwon, Kim, Kim, Hwang and Seong. This is an open-access article distributed under the terms of the Creative Commons Attribution License (CC BY). The use, distribution or reproduction in other forums is permitted, provided the original author(s) and the copyright owner(s) are credited and that the original publication in this journal is cited, in accordance with accepted academic practice. No use, distribution or reproduction is permitted which does not comply with these terms. 